\title{
Sequências volumétricas por ressonância magnética em tumores da cabeça*
}

\section{Volumetric magnetic resonance sequence in head tumors}

Ronaldo Luiz dos Santos ${ }^{1}$ Bergman Nelson Sanchez Munhoz ${ }^{2}$ Rafael Eidi Goto ${ }^{3}$ Homero Jose de Farias e Melo ${ }^{4}$ Leandro Nobeschi ${ }^{5}$
* Recebido em: 02/08/2016. Aprovado em: 03/11/2017.

1 Pós-graduando em Tomografia Computadorizada e Ressonância Magnética. Faculdade de Ciências Médicas da Santa Casa de São Paulo (FCMSCSP). São Paulo-Brasil.

2 Docente da Faculdade de Ciências Médicas da Santa Casa de São Paulo (FCMSCSP) e da Universidade Nove de Julho (UNINOVE). São Paulo-Brasil.

3 Docente da Faculdade de Ciências Médicas da Santa Casa de São Paulo (FCMSCSP). São Paulo-Brasil.

4 Faculdade de Ciências Médicas da Santa Casa de São Paulo (FCMSCSP) e Centro Universitário São Camilo. São Paulo-Brasil.

5 Faculdade de Ciências Médicas da Santa Casa de São Paulo (FCMSCSP), Universidade de Santo Amaro (UNISA) e Faculdades Metropolitanas Unidas (FMU). São Paulo-Brasil. E-mail:ln.nobeschi@gmail.com.

\section{Resumo}

A aplicação das sequências de volumétricas por Ressonância Magnética - VIBE (Volume Interpolated Breath Hold Examination) é utilizada para verificação do estadiamento de tumores da cabeça, e demonstram ser o melhor método nesses casos devido ao menor tempo de aquisição. Este artigo visa elucidar a aplicação e a relevância das sequências de volumétricas por meio de uma revisão da literatura, analisando a aquisição por meio do método VIBE, apurando as vantagens com relação ao T1 volumétrico e as demais sequências, T2, Fast Spin Eco e Spin Eco, pós-contraste com relação ao tempo. O exame VIBE equivale a um mecanismo relativamente novo de sequência na ressonância magnética (RM) gradiente-eco, sendo capaz de encurtar tempos de aquisição. Para essa hipótese, demonstra-se ser de grande valia em imaginologia do cérebro, como um substituto para imagens pesadas em T1 spin-eco póscontraste convencional na avaliação dos tumores da cabeça.

Palavras-chave: Ressonância Magnética. Tumores. Sequência Volumétrica. Contraste.

\begin{abstract}
The application of volumetric sequences for MRI - VIBE (Volume Interpolated Breath Hold Examination) are used to verify the staging of head and neck tumors, and prove to be the best method in these cases due to the shorter acquisition time. This article aims to clarify the application and relevance of volumetric sequences through a literature review, analyzing acquisition through VIBE method, calculating the advantages with respect to the volumetric T1 and other sequences, T2, Fast Spin Echo and Spin Eco, post-contrast with respect to time. The Vibe take equals a relatively new mechanism of gradient-echo MRI sequence, being able to shorten acquisition time. For this hypothesis it demonstrates to be very useful in imaging the brain, as a substitute for heavy images in conventional post-contrast spin-echo T1 in the evaluation of tumors of the head and neck.
\end{abstract}

Keywords: MRI. Tumors. Volumetric Sequence. Contrast. 


\section{Introdução}

Os tumores da cabeça no âmbito mundial equivalem a $10 \%$ dos tumores malignos. Envolvem várias situações, cerca de $40 \%$ dos casos ocorrem na cavidade oral, $25 \%$ na laringe, $15 \%$ na faringe, $7 \%$ nas glândulas salivares e $13 \%$ nos demais locais. Anualmente, ocorrem mais de oito milhões de novos casos de câncer no mundo, dos quais 212.000 originam-se na boca. No Brasil o Instituto Nacional de Câncer estimou, para o ano de 2002, a ocorrência de 337.535 casos novos de câncer, dos quais 11.255 de boca (3,3\%) (CARDOSO et al., 2005).

Em nosso país, o câncer representa a terceira principal causa de morte. A cirurgia, radioterapia (RT) continuam sendo os principais e mais eficazes métodos de tratamento dos tumores da cabeça bem como a quimioterapia (QT). As principais causas desse acometimento justificam-se devido à má alimentação, tabagismo, álcool, fatores químicos e inclusive a falta de conhecimento e ainda por não terem um acompanhamento médico (CARDOSO et al., 2005).

A Tomografia Computadorizada (TC) ajuda no diagnóstico preciso do tumor no comprometimento ósseo local, sendo o melhor método para identificar estágios iniciais de erosão óssea. A Ressonância Magnética (RM) vem sendo utilizada conjuntamente à TC. As principais vantagens, com relação a ressonância, correspondem a melhor definição das margens tumorais juntamente aos tecidos moles adjacentes. Como a melhor caracterização da invasão tumoral óssea se promove por intermédio da TC incluindo-se a boa avaliação da extensão tumoral, um correto e fidedigno estadiamento, será promovido por meio da RM. Em comparação à TC, prevê uma melhor visualização da metástase quando não se tem um único tecido acometido pelo câncer, contudo, ambas se tornam importantes para um bom diagnostico (GONZALES et al., 2005).

O realce por contraste é extremamente valioso em muitos processos patológicos, inclusive tumores, inflamações e possíveis infecções. Embora essas patologias contenham um grau elevado de água e frequentemente, sejam visualizadas em imagens ponderadas em T2, às vezes o contraste entre a lesão e o tecido circundante é insuficiente. Além disso, as imagens ponderadas em T1 evidenciam uma relação sinal/ ruído mais elevada sendo, portanto, vantajosas; entretanto, a água e a doença com frequência são isointensas e nessas sequências e, às vezes, é necessário realçar, seletivamente, a doença pela administração de meios de contraste. Isso pode ser feito indiretamente, por via intravenosa (IV), oral ou retal, diretamente, no caso de uma estrutura como uma articulação (WESTBROOK, 2010).

Existem dois tipos de meios de contraste: os que produzem o contraste positivo e os que resultam em contraste negativo. $\mathrm{O}$ meio de contraste mais positivo usado em RM é o gadolínio (Gd). O gadolínio é uma substância paramagnética, com um momento magnético relativamente amplo (WESTBROOK, 2010).

As sequências de pulso que podem ser aplicadas: T1, T2, Spin eco (SE), Fast spin eco (FSE), a volume interpolated breath-hold examination (VIBE) que pode substituir o T1 pós contraste ${ }^{3}$. De acordo com os artigos, a sequência T1 volumétrica é uma imagem pesada e a mesma pode se degradar por artefatos de movimentos e seu tempo de aquisição acaba sendo longo, por mais que o profissional mude os parâmetros para ganhar tempo, pode ocorrer uma perda na qualidade da imagem. A sequência VIBE, por sua vez, o seu tempo é menor e permite a reconstrução nos três planos: sagital, axial e coronal, sem perda da resolução da imagem (WU et al., 2014).

$\mathrm{Na}$ imagem em T1 spin-eco ou turbo spin-eco (TSE), sequências com supressão de gordura tradicional (imagens em T1); podem se degradar por movimento e artefatos de pulsação em estudos da cabeça. Nosso propósito é avaliar o papel de uma sequência spin-eco ponderada em T1 com supressão de gordura (O exame de Apneia volumétrica interpolados - VIBE) na cabeça, em comparação com o padrão de imagens ponderadas em T1 (WU et al., 2014).

A tomografia computadorizada e ressonância magnética são aceitas, amplamente, no diagnóstico e estadiamento dos tumores da cabeça. Em geral as imagens por ressonância magnética, por sua tissularudade e definição anatômica, são superiores às de TC, em especial no que tange à obtenção de um contraste no excepcional tecido, o que contribui para a delimitar extensão do tumor. Embora T1 sem contraste e imagens de RM ponderadas em T2 possam delinear tumores, a administração de material de contraste é recomendada porque a intensidade do sinal de tumores aumenta nas imagens ponderadas em T1 pós-contraste, o que resulta em melhor delineamento de tumores contra os músculos e melhor representação de dimensão tumoral ou invasão perineural. Apesar dessa vantagem da RM, o tempo de digitalização relativa- 
mente longa (vários minutos por sequência com spin-eco tradicional ou técnica de fast spin-eco) gera a perda de qualidade de imagem devido a artefatos de movimento na cabeça região. Além disso, essa degradação da ressonância magnética tem sido um obstáculo na realização de estudos dinâmicos com injeção rápida de agentes de contraste (WESTBROOK, 2010).

O exame de apneia volumétrico interpolado (VIBE) é uma sequência por RM a qual o gradiente-eco proporciona tempos de aquisição curtos, sem degradar a qualidade da imagem. Essa sequência permite a geração de imagens pesadas em T1 com supressão de gordura. Características do sinal de sequências podem ser semelhantes aos de gradiente-eco convencional ou de baixo ângulo filmados sequências de eco de gradiente. Alguns estudos já revelaram que a sequência VIBE fornece imagens comparáveis ou superiores aos obtidos utilizando sequências de eco-gradiente e, portanto, a sequência VIBE é útil para o estudo abdominal, cérebro e do tórax. A hipótese é que essa técnica de varredura rápida pode produzir imagens na região da cabeça com qualidade de imagem que permeia o laudo e por conseguinte o diagnóstico (WESTBROOK, 2010).

Este artigo tem por objetivo fazer uma análise com a aquisição VIBE mostrando os seus pontos fortes com relação ao $\mathrm{T} 1$ volumétrico e as demais sequências, T2, Fast Spin Eco e Spin Eco, pós contraste com relação ao tempo.

\section{Método}

Trata-se de um estudo de revisão da literatura. Para compilar os artigos, utilizamos a base de dados da SciELO e PubMed. O período da consulta na base de dados foi abril a junho de 2016. Os descritores utilizados para a pesquisa nas bases de dados foram: ressonância magnética, tumores e sequência volumétrica. Foram incluídos, apenas, artigos em seres humanos, nos idiomas: português, inglês e espanhol. Após a leitura dos resumos e periódicos completos excluímos os artigos que não trataram da proposta relacionada aos nossos objetivos.

\section{Principais resultados obtidos a partir da re- visão da literatura}

Na eventualidade de apresentação tumoral, localizada na cabeça, a caracterização típica no exame equivale a uma anomalia, diferenciando os tecidos normais dos acometidos pelo tumor. A RM nos trará um pré-diagnostico, reiterando que apenas a biopsia será determinante para a classificação quanto à benignidade e/ou malignidade (WESTBROOK, 2010).

Embora as imagens da região da cabeça pareçam menos críticas, à primeira vista, os artefatos de movimentos graves ocorrem com bastante frequência em exames de rotina. Protocolos convencionais incluem, geralmente, sequências T1, TSE, que são especialmente sensíveis ao movimento e fluxo. Se os pacientes não são capazes de suprimir a deglutição ou tosse durante a aquisição, as imagens são processadas no diagnóstico. Por outro lado, o exame adequado da região da cabeça, no modo VIBE, se apresenta como uma alternativa promissora para exames dessa região. Graças a essa aplicação, os efeitos provenientes do movimento, como a deglutição, afetam pouco a qualidade do exame. A sequência, também, mantém uma sensibilidade convincente para lesões torácicas na presença de movimentos respiratórios. O modo VIBE torna-se imune ao efeito fantasma de artefatos, nesse contexto, os exames podem ser realizados com resolução espacial isotrópica de altura, o que permite uma reconstrução retrospectiva em múltiplos planos (MPRs). Dessa forma, é possível substituir T1 múltiplo convencional (BLOCK et al., 2013).

A principal vantagem da imagem $3 \mathrm{D}$ é a capacidade de adquirir dados com resolução aproximadamente isotrópica. Isso permite a reformatação multiplanar, que simplifica protocolos de imagem e reduz o tempo de medição quando imagens em múltiplos planos é necessária. Essas vantagens são demonstradas em vários estudos. Embora, em princípio, a resolução isotrópica possa ser adquirida com sequências $2 \mathrm{D}$, isto requer longos comprimentos de pulsos de RF que aumentam o tempo de aquisição. Além disso, as seções estreitas são, muitas vezes, indisponíveis com sequências 2D, especialmente em sistemas mais antigos. Gradiente eco rápido preparado por magnetização (MP RAGE) é uma sequência em 3D que permite a aquisição de conjuntos de dados $3 \mathrm{D}$ em um prazo razoável, oferecendo excelente contraste (BLOCK et al., 2013).

A alta-resolução espacial ponderadas em T1 conjuntos de dados 3D do cérebro pode, também, ser efetivamente obtida com VIBE. Essa técnica, quando configurado para exigir o mesmo tempo de imagem como MP RAGE, demonstra características de contraste semelhan- 
tes ou ligeiramente melhores e menos artefatos. Embora ambas as técnicas utilizem sequências Gradiente Eco (GRE) ultracurtos TRs e ambas sejam métodos em T1, os mecanismos de contraste diferem. Com MP RAGE, o contraste é desenvolvido pela aplicação de um seletivo $180^{\circ}$ de pulso de inversão antes da aquisição rápida GRE. As características de contraste dessa sequência dependem, entre outros parâmetros, da T1 que é eficaz sobre o comprimento do período de recuperação de magnetização. Como o contraste é desenvolvido de forma similar nas sequências de GRE e TR, o ângulo de aleta se torna importante (BLOCK et al., 2013)

Com VIBE, o aumento da eficiência de imagem é atingido por amostragem assimétrica do espaço $\mathrm{K}$ e de zero-enchimento na direção seção. Zero-enchimento é um método de interpolação que se tornou uma forma consagrada para reduzir artefatos de volume parcial em 3D. Em combinação com amostragem assimétrica, de zero-enchimento dá uma melhor resolução espacial. A relação entre a assimetria de amostragem e resolução espacial é consideravelmente complexa (BLOCK et al., 2013).

A VIBE é uma sequência eficiente em que as amostras de dados são adquiridas continuamente durante o período de criação de imagens. Com MP RAGE, aproximadamente metade do tempo de aquisição é necessária para a preparação e recuperação de magnetização, durante o qual não são adquiridos. Assim, dois sinais podem ser obtidos e média com VIBE no tempo para a aquisição utilizando MP RAGE. Isso explica, em parte, por que relação sinal ruído (RSR) e relação contraste ruído (RCR) em imagens VIBE são melhores do que aquelas em imagens MP RAGE. Por outro lado, os tempos de imagem podem ser diminuídos ou a matriz de amostras pode ser aumentada sem que seja aumentado, também, o tempo de imagiologia por média, embora essas alterações ocorram em detrimento do RSR e RCR (BLOCK et al., 2013).

Em medições fantasmas, descobrimos que VIBE oferecia melhores RSR do que fizeram RAGE MP para tempos de relaxamento T1 que eram mais curtos, em seguida, $600 \mathrm{~ms}$, que é um pouco abaixo do valor de T1 para a matéria cerebral branca (ou seja, $800 \mathrm{~ms}$ ). Mais importante, os resultados revelaram que o fantasma declive da curva foi mais acentuado do que para IMPRESSÃO MP para RAGE, o que implica que deverá ser maior contraste para lesões de aumento de contraste (WETZEL et al., 2002)

O tempo de aquisição para a sequência T1 era, apenas, metade das sequências realizadas GRE, dobrando o número de médias para a sequência SE para dar tempos iguais de imagem iria melhorar tanto RSR e RCR. No entanto, na prática clínica, imagens ponderadas em T1 pós-contraste, normalmente, devem ser adquiridas em mais de um plano. Pelo menos duas aquisições separadas são, portanto, necessárias para a sequência SE T1-ponderadas, mas apenas uma é necessária para VIBE e sequências de RAGE MP. Para os casos em que é necessário, apenas, um plano, a média sinal melhoraria SE RSR e RCR proporcionalmente. Igualmente, quando são necessários três planos, SE RSR e RCR sofreria em relação aos das sequências GRE quando normalizado para o tempo de aquisição (WETZEL et al., 2002)

Os requisitos mais importantes para VIBE correspondem a: supressão de gordura uniforme, excelente tecido contraste, nitidez da imagem, alguns artefatos, e tempo de varredura curto. As novas funcionalidades incluem um novo reordenamento do espaço $\mathrm{K}$, um novo regime de supressão de gordura, e uma nova funcionalidade de reconstrução (WETZEL et al., 2002; MAZZOLA, 2009).

Os tumores da cabeça são doenças tardias e prevalecem sem acometimentos associados. Parte da população acometida por tumores não tem esclarecimento e conhecimento adequado sobre o assunto, dessa forma, não buscam em tempo hábil profissionais da saúde para que seja realizado o diagnóstico e terapêutica. A maior parte dos pacientes recorrem ao SUS (Sistema Único de Saúde), apresentando um comprometimento significativo pelo avanço do tumor. Dessa maneira, o tratamento tende a perder a eficácia. No que diz respeito aos sistemas públicos de saúde, a morosidade no primeiro atendimento e as demais necessidades, incluindo-se, principalmente, os exames de imagem são determinantes na sobrevida dos pacientes/clientes (AMAR et al., 2002).

A TC e RM promovem um ótimo diagnostico de doenças, principalmente os tumores, salientando que, por sua vez, a RM corresponde ao melhor método de detecção/diagnóstico para esse tipo de tumor da cabeça, por se tratar de partes moles e estadiamento (AMAR et al., 2002).

A análise promovida pela comunidade médica constata que a aquisição VIBE detém significativa relevância em comparação à sequência T1 volumétrica pós-contraste, em virtude dos benefícios recorrentes ao tempo de exame e pela sensibilidade associada ao movimento na detecção dos tumores da cabeça (BOTTOMLEY et al., 
1984; MUGLER; BROOKEMAN, 1990).

A reconstrução da VIBE, nos 3 planos sagital, axial e coronal, ocorre sem perder a sua alta resolução. O T1 não nos permite esse resultado, pois, quando se alteram os parâmetros da imagem, há uma perda em sua resolução podendo, assim, comprometer o diagnóstico. O tempo da aquisição VIBE é menor, trazendo, assim, melhor conforto ao paciente com um diagnóstico preciso sem perda de qualidade e resultados nos achados da imagem (figuras 01 e 02) (CHANDARANA et al., 2011).

Figura 1 - Imagens de RM em um paciente com hipótese de doença metastática localizada na fossa posterior do crânio. Imagem A ponderada em T1: indica formação de artefatos de pulsação, pela sobreposição do plexo coroide do quarto ventrículo, semelhante a uma possível lesão realçada por contraste (seta). Imagem B: demonstra consideráveis artefatos fantasmas (setas) prejudicando a visualização da ponte e cerebelo. Imagem C: VIBE não mostra nenhum artefato.
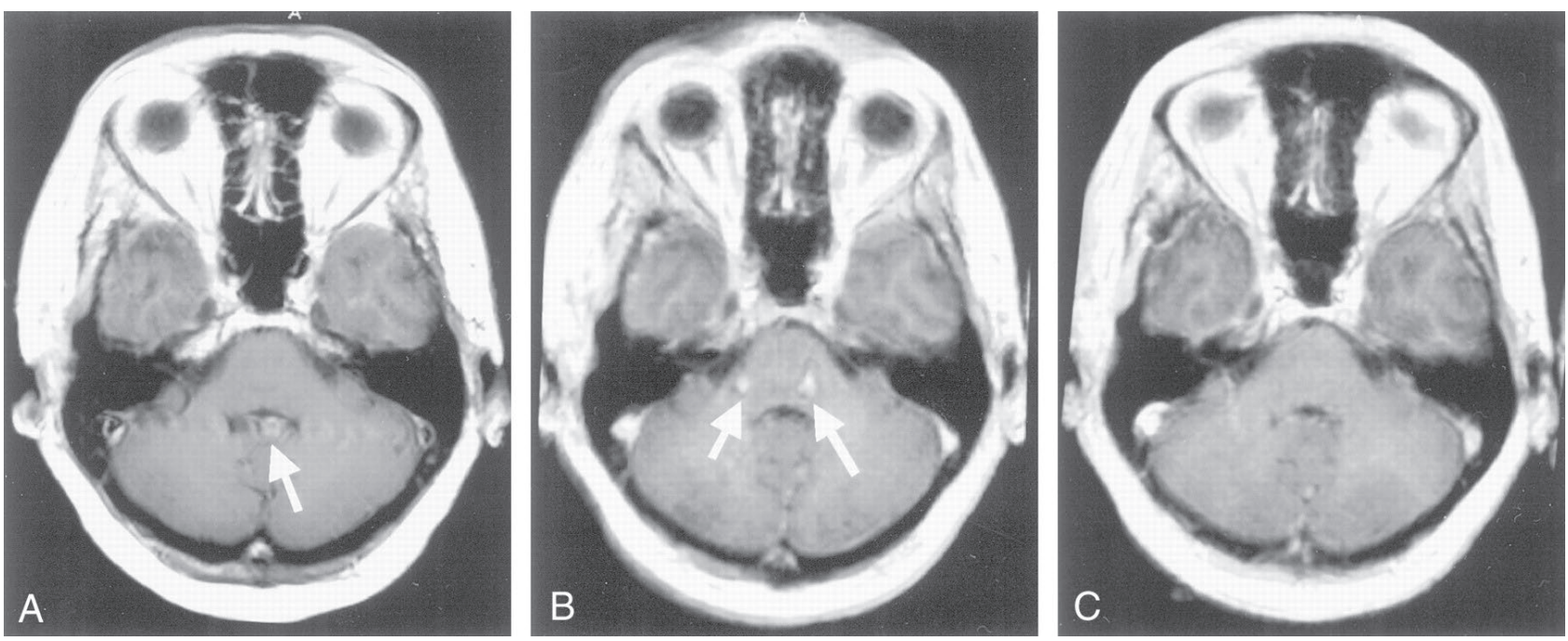

Fonte: STEPHAN et al. (2002).

Figura 2 - Linfoma cerebral primário. Imagens A, B e C com lesão realçada por contraste, (seta em A) é bem visualizado tanto na imagem T1 (A) e a reforma VIBE $5 \mathrm{~mm}$ (B) , mas é menos óbvio na RAGE MP $5 \mathrm{~mm}$ reforma (C).
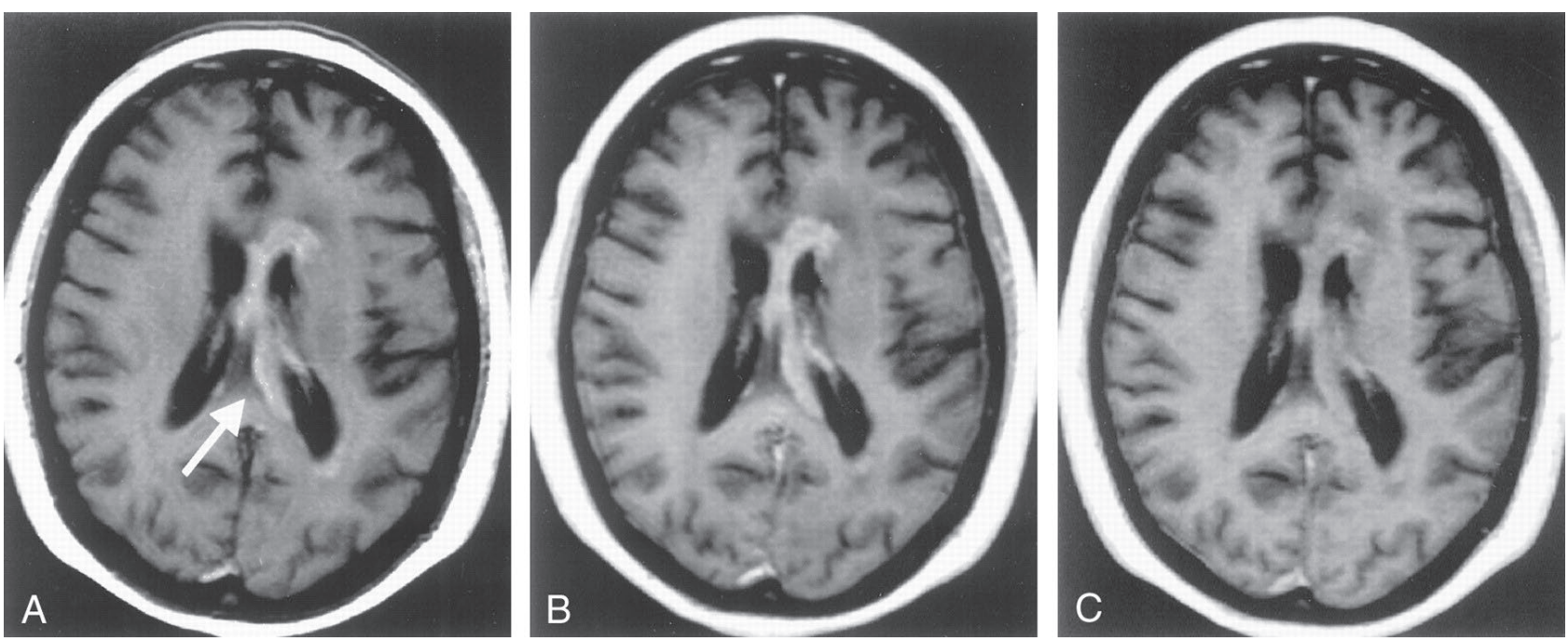

Fonte: STEPHAN et al. (2002).

\section{Considerações finais}

As sequências de exames T1 volumétrico, T2, Fast Spin Eco e Spin Eco são utilizadas na rotina para exames da cabeça e pescoço, principalmente com hipóteses diagnósticas de tumores. Contudo, observamos que o protocolo no modo VIBE permite o diagnóstico de lesões tu- morais com uma alta sensibilidade, excelente qualidade de imagem, definição de contraste, supressão de gordura uniforme, menor tempo de execução do exame, reconstrução 3D e diminuição significativa nos artefatos fantasmas, gerados por movimentos de deglutição, tosse e respiração, em comparação aos exames de rotina. Assim, 
o modo VIBE permite identificar, de forma precisa, a área tumoral, separando-a do tecido saudável, o que auxilia o diagnóstico preciso e o planejamento para a terapêutica mais indicada.

\section{Referências}

AMAR, A. et al. Qualidade de vida e prognóstico nos carcinomas epidermóides de cabeça e pescoço. Revista Brasileira de Otorrinolaringologia, São Paulo, v. 68, n. 3, p. 400-403, maio 2002.

BLOCK, T. K. et al. Improving the Robustness of Clinical T1-Weighted MRI Using Radial VIBE. Clinical Head to Toe Imaging. New York, USA, 2013. Disponível em: <www.siemens.com/magnetom-word>. Acesso em: 17 nov. 2017.

BOTTOMLEY, P. A. et al. A review of normal tissue hydrogen NMR relaxation times and relaxation mechanisms from 1-100 MHz: dependence on tissue type, NMR frequency, temperature, species, excision, and age. Medical Physics, Hoboken, v. 11, p. 425-448, jul./aug. 1984.

CARDOSO, M. F. A. et al. Prevenção e controle das sequelas bucais em pacientes irradiados por tumores de cabeça e pescoço. Radiologia Brasileira, São Paulo, v. 38, n. 2, p. 107-115, mar./apr. 2005.

CHANDARANA, H. et al. Free-breathing radial 3D fat-suppressed T1-weighted gradient echo sequence: a viable alternative for contrast-enhanced liver imaging in patients unable to suspend respiration. Investigative Radiology, Hagerstown, v. 46, n. 10, p. 64853, oct. 2011.
GONZALEZ, F. M. et al. Carcinoma espinocelular do conduto auditivo externo: estudo de tomografia por seis casos. Radiologia Brasileira, São Paulo, v. 38, n. 3, p. 181-185, may/jun. 2005.

MAZZOLA, E. A. Ressonância magnética: princípios de formação da imagem e aplicações em imagem functional. Revista Brasileira de Física Médica, Porto Alegre, v. 3, n. 1, p. 117-129, jul./dez. 2009.

MUGLER, J. P.; BROOKMAN, J. R. Three-dimensional magnetization prepared rapid gradient-echo imaging (3D MP RAGE). Magnetic Resonance in Medicine, New York, v. 15, p. 152-157, jul. 1990.

STEPHAN, G. et al. Three-dimensional, T1- three-dimensional, T1-weighted gradient-echo imaging of the brain with a volumetric interpolated examination. AJNR American Journal of Neuroradiology, Oak Brook, v. 23, p. 995-1002, jun./jul. 2002.

WESTBROOK, C. Manual de técnicas de ressonãncia magnética. 3. ed. Rio de Janeiro: Guanabara Koogan, 2010 .

WETZEL, G. S. et al. Three-Dimensional, T1-Weighted gradient-echo imaging of the brain with a volumetric interpolated examination. AJNR American Journal of Neuroradiology, Oak Brook, v. 23, n. 6, p. 995-1002, jun./jul. 2002.

WU, X. et al. Contrast-enhanced radial 3D fat-suppressed T1-weighted gradient-recalled echo sequence versus conventional fat-suppressed contrast-enhanced T1-weighted studies of the head and neck. AJR American Journal of Roentgenology, Leesburg, v. 203, n. 4, p. 883-889, oct. 2014. 